\title{
Estudio de la variación de algunas magnitudes reológicas, ópticas y térmicas de un aceite de oliva refinado termooxidado
}

\author{
Por J. Cabrera Martín \\ Instituto de la Grasa y sus Derivados. Avda. Padre Garcia Tejero, 4 \\ 41012 - Sevilla.
}

\section{RESUMEN}

Estudio de la variación de algunas magnitudes reológicas, ópticas y térmicas de un aceite de oliva refinado termooxidado.

Al calentar un aceite en presencia de oxígeno, se producen una serie de reacciones que van a motivar la aparición de nuevos componentes, los cuales van a ocasionar variaciones en sus magnitudes físicas.

El aceite que se ha utilizado en este estudio ha sido uno de oliva refinado, el cual se ha calentado a una temperatura de $180^{\circ} \mathrm{C}$, hasta un limite de 72 horas.

Se ha determinado la concentración de triglicéridos no alterados por cromatografía en columna de sílice, calculando posteriormente la fracción molar correspondiente a cada tiempo de calentamiento.

Se han medido, el volumen específico, la viscosidad dinámica y el indice de refracción, ajustando estos valores a las ecuaciones que relacionan estas magnitudes con la temperatura.

Así mismo, también se ha medido la entalpia especifica y la temperatura de fusión.

Por último, se ha estudiado la variación que experimentan los parámetros que definen esas ecuaciones, con la alteración producida en el aceite.

PALABRAS-CLAVE: Aceite de oliva - Grasa calentada - Propiedades físicas (variación).

\section{SUMMARY}

Study of some changes in the rheologic, optic and thermic magnitudes of a refined and thermooxidized olive oil.

When heating an oil in the presence of oxygen, several reactions were produced, which caused some changes in its physical properties.

The oil used in this study was heated at $180^{\circ} \mathrm{C}$ for different periods of time until a maximum of 72 hours.

The concentration of unaltered triglycerides was determined by silica column chromatography, and the molar fraction corresponding to each heating period was calculated.

The specific volume, the dynamic viscocity and the refractive index were measured, and the obtained values were adjusted to the corresponding equations that relate these magnitudes with temperature.

The specific enthalpy and the melting point were also measured.

Finally, the changes experienced by the measured parameters, defined in the above equations were discussed in relation to the changes produced in the oil.

KEY-WORDS: Heated fat - Olive oil - Physical properties (variation).

\section{INTRODUCCION}

Al calentar un aceite en presencia de oxígeno, se producen una serie de reacciones que van a motivar la aparición de una gran cantidad de compuestos nuevos (1) los cuales pueden resumirse en: a) Productos volátiles, como hidrocarburos, aldehidos, cetonas, ácidos grasos cortos, ésteres, lactonas, ...

b) Compuestos oxigenados monómeros, como ácidos grasos libres, hidroxiácidos, oxiácidos, hidroperóxidos, ...

c) Compuestos cíclicos, alicíclicos y aromáticos.

d) Dímeros y polímeros en general.

La mayor o menor concentración de estos compuestos de alteración depende fundamentalmente de la temperatura y del tiempo de calentamiento. Así como de la presencia en mayor o menor cantidad de oxígeno, que favorezca el desarrollo de reacciones oxidativas. La presencia de humedad, por otra parte, da lugar a reacciones de hidrólisis. Por último, la presencia de metales prooxidantes en el recipiente que contiene el aceite influye directamente en su degradación al favorecer las reacciones de oxidación.

La variación que la presencia de todos estos compuestos en la composición del aceite va a producir variaciones en las magnitudes físicas de éste, como son:

a) Aumento de la densidad, como consecuencia de la incorporación del oxígeno de oxidación.

b) Aumento de la viscosidad y de la tendencia a la espumación, como consecuencia de la formación de polímeros.

c) Incremento del índice de refracción y de la absorción ultravioleta, debido a la conjugación de dobles enlaces y a la acumulación de productos oxigenados.

d) Disminución del punto de fusión, debido a la aparición de los productos de descomposición.

En este trabajo se van a estudiar las variaciones de algunas de estas magnitudes en función de la alteración que estos compuestos producen en el aceite, al ser calentados en presencia de oxígeno.

\section{PARTE EXPERIMENTAL}

\subsection{Preparación de las muestras}

El aceite que ha servido de base para este estudio ha sido uno de oliva refinado, su densidad a $20^{\circ} \mathrm{C}$ tiene un valor de $0,9132 \mathrm{~g} / \mathrm{mL}$, y su cromatografía de ácidos grasos ha dado, en lo que se refiere a componentes mayorita- 
rios, la siguiente composición: ácido palmítico 11,3\%, ácido palmitoleico $1,5 \%$, ácido esteárico $2,0 \%$, ácido oleico $77,6 \%$ y ácido linoleico $7,6 \%$.

Este aceite se ha calentado en presencia de aire, lavado y seco, a $180^{\circ} \mathrm{C}$, en frascos de $500 \mathrm{~mL}$, que contenían $250 \mathrm{~mL}$ de aceite y en los que se le ha hecho borbotear un flujo de aire de 3 litros por hora a la presión atmosférica. Dichos frascos estaban colocados en un baño termostático. La precisión de la temperatura del baño es de $\pm 5^{\circ} \mathrm{C}$

Este aceite se ha calentado durante distintos tiempos hasta un límite de 72 horas. Simultáneamente y en el mismo baño se han puesto dos frascos, formándose así dos series de muestras independientes.

En las figuras, que se muestran posteriormente, sobre relaciones de las diferentes magnitudes medidas, las correspondientes a la primera serie se representan por triángulos y las de la segunda por cuadrados. Las pequeñas diferencias que aparecen entre ambas series es debido a algún pequeño desequilibrio en el flujo de aire, puesto que la temperatura y el tiempo de calentamiento fueron los mismos.

\subsection{Aparatos utilizados y técnicas de cálculo}

a) Determinación de la alteración del aceite.- La concentración de triglicéridos no alterados, que en lo que sigue se les designará por TNA, se ha obtenido por cromatografía en columna de sílice según el método recomendado por la IUPAC (2), separando solo la parte no polar. Los productos de descomposición se han obtenido por diferencia.

b) Medida de densidad.- Para su determinación se han utilizado picnómetros de $25 \mathrm{~mL}$, previamente calibrados con agua destilada.

c) Medida de viscosidad.- Previamente a las medidas de viscosidad se ha comprobado el carácter reológico newtoniano de todas las muestras, con un reómetro "Rheomat 15" de "Contraves".

Las medidas de viscosidad se han hecho con un viscosímetro de caída de bola, "Hoepple, tipo BH2".

d) Medida del índice de refracción.- Estas medidas se han hecho con un refractómetro "Hilder", utilizando para la medida luz natural.

e) Medidas de temperatura y termostatación.- Tanto las medidas de densidad y viscosidad, como las de índice de refracción se han realizado con un termostato "Colora", en un intervalo de temperaturas comprendido entre 5 y $95^{\circ} \mathrm{C}$, en las medidas de índice de refracción el intervalo ha sido de 10 a $70^{\circ} \mathrm{C}$, con una precisión de \pm $0,2^{\circ} \mathrm{C}$.

Las temperaturas se han medido con termómetros de mercurio graduados en décimas de grado.

f) Medidas térmicas. - Las medidas térmicas se han hecho con un calorímetro diferencial de barrido, tipo DSC4 de "Perkin-Elmer".

g) Unidades en que se dan las medidas y error del que van afectadas.- El error de las determinaciones de la concentración de "TNA" es menor que el cinco por ciento.
Las medidas de volumen específico (obtenidas por el inverso de la densidad) se dan en mililitros por gramos, y su error es menor del cinco por mil.

Las medidas de viscosidad dinámica se expresan en milipascales por segundo y el error del que van afectadas es menor que el cuatro por ciento.

El error de las medidas de índice de refracción es menor que el cuatro en diez mil.

Las medidas de entalpía específica de fusión se dan en calorías por gramo y el error del que van afectadas es del dos por ciento. Las temperaturas se dan en grados Kelvin, salvo que se indique en grados centígrados.

Los tiempos de calentamiento se han medido en horas.

h) Ajustes y precisión de los mismos. - Las distintas relaciones entre las magnitudes medidas, se han ajustado por "mínimos cuadrados", comprobándose su bondad mediante el "tanto por ciento de error standard" "SPE" ("standard percentage error", según la literatura americana), definido por

$$
S P E=100\left[\sum\left(\frac{X_{c a l}-X_{m e d}}{X_{m e d}}\right)^{2} / N\right]^{\frac{1}{2}}
$$

donde $X_{c a \mid}$, es el valor de la magnitud calculado a través del ajuste, $X_{\text {med }}$, es el valor de la misma medido y $\mathrm{N}$ el número de medidas.

Como se observa, el SPE es el error relativo cuadrático medio del ajuste.

\section{MEDIDAS}

\section{a) Medida de la alteración del aceite}

Es norma común en estudios sobre líquidos de "calidad técnica", definir el peso molar medio de éstos, a partir de los pesos moleculares de los componentes mayoritarios, aplicando la fórmula

$$
P=\sum f_{i} P_{i}
$$

donde $f_{i}$ es la fracción molar del componente $i$, cuyo peso molecular es $P$.

En este estudio no se ha averiguado la composición en triglicéridos que constituyen el aceite utilizado. Por otra parte, tampoco se conocen los productos a los que ha dado lugar la descomposición del mismo, por lo que no se puede calcular el peso molar según la fórmula anterior. Se va a suponer, sin embargo, que se puede definir un peso molar medio para el aceite, y también, que se puede definir otro peso molar medio de los productos de descomposición, que se mantendrá constante al menos, dentro del intervalo de tiempo en el que se ha calentado el aceite.

Admitidos estos supuestos, la fracción molar de los TNA tiene por expresión

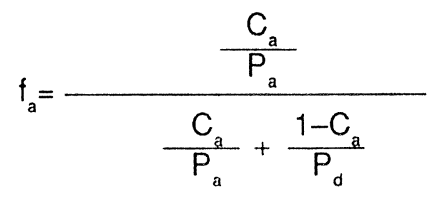


donde $\mathrm{C}_{\mathrm{a}}$ y $\mathrm{P}_{\mathrm{a}}$ son la concentración y el peso molar de los TNA y $\mathrm{P}_{d}$ el peso molar de los productos de descomposición. tiene

Multiplicando numerador y denominador por $\mathrm{P}_{\mathrm{a}}$, se

$$
f_{a}=\frac{C_{a}}{C_{a}+\frac{1-C_{a}}{N}}
$$

donde se ha llamado $N$ al cociente $P_{\delta} / P_{a}$. Número que es superior a la unidad, al existir polimerización entre otros productos de descomposición del aceite.

Suponer que, tanto el aceite como los productos de descomposición, son una sola sustancia aunque de naturaleza compleja, con un peso molar medio definido, lleva a poder expresar la descomposición térmica del aceite, en presencia de oxígeno, mediante la siguiente reacción

Aceite + Oxígeno $\stackrel{\text { calor }}{\rightarrow}$ Productos de descomposición

cuya evolución en función del tiempo seguirá una determinada cinética, definida por la ecuación

$$
\frac{d\left(f_{a}\right)}{d t}=K\left(f_{a}\right)^{n}
$$

siendo $\mathrm{K}$ la constante de velocidad y $\mathrm{n}$ el orden de evolución de la descomposición.

Como las concentraciones de los TNA se han medido después de haber calentado el aceite durante distintos tiempos, el método a seguir para calcular la cinética de descomposición es el de integración. Separando variables en la ecuación anterior, e integrando, se tiene

$$
\int_{f_{a}}^{f_{a}} \frac{d\left(f_{a}\right)}{f_{a}^{n}}=k \int_{0}^{t} d t
$$

Según sea el valor de n, la ecuación cinética tomará las formas siguientes:

Si $n=0$, la ecuación cinética será la recta

$$
f_{a}=f_{a o}+K t
$$

Si $n=1$, la ecuación cinética será la exponencial.

$$
f_{a}=f_{a o} \exp (K t)
$$

Si $n>1$, la ecuación cinética será la ecuación

$$
\frac{1}{f_{a}^{n-1}}=\frac{1}{f_{a 0}^{n-1}}-(n-1) K t
$$

donde $f_{a 0}$ es la fracción molar de los TNA antes de empezar el calentamiento, es decir, los del aceite original (valor muy próximo a la unidad).

Salvo en el caso $n=0$, las ecuaciones cinéticas no son rectas, sin embargo, se pueden linealizar, tomando logaritmos, en el caso $n=1$, o cambiando la variable $f_{a^{\prime}}^{n-1}$ por su inversa en el caso $n>1$.

El parámetro que define una mayor o menor linealidad de una serie de puntos alineados, en un ajuste por "mínimos cuadrados", es su coeficiente de correlación. Cuanto más se acerque a la unidad más lineal será.
Así planteado el problema, se ha calculado siguiendo un proceso iterativo el coeficiente de correlación de la fracción molar de los TNA en función del tiempo, dando sucesivos valores a $\mathrm{N}$, a partir de la unidad, dentro de cada orden de evolución, con la ecuación linealizada.

Se ha encontrado que para $N=1,51$ y $n=1$, el coeficiente de correlación es el máximo que se puede obtener, entre todos los órdenes, siendo este valor 0,9990 .

Introducido el valor de $\mathrm{N}$ encontrado, en la fórmula (2) que define la fracción de los TNA, se han calculado

\begin{tabular}{|c|c|c|c|c|c|c|}
\hline \multirow[b]{2}{*}{$\mathbf{t}$} & \multicolumn{3}{|c|}{ Serie 1} & \multicolumn{3}{|c|}{ Serie 2} \\
\hline & $C_{a}$ & $f_{a}$ & $\mathbf{P}_{r}$ & $c_{a}$ & $f_{a}$ & $P_{r}$ \\
\hline 0 & 0,984 & 0,989 & 1,0056 & 0,992 & 0,995 & 1,0026 \\
\hline 2,5 & 0,959 & 0,972 & 1,0143 & 0,941 & 0,960 & 1,0204 \\
\hline 5 & 0,927 & 0,950 & 1,0255 & 0,936 & 0,957 & 1,0219 \\
\hline 8 & 0,900 & 0,931 & 1,0352 & 0,891 & 0,925 & 1,0383 \\
\hline 16 & 0,825 & 0,877 & 1,0627 & 0,801 & 0,859 & 1,0719 \\
\hline 24 & 0,729 & 0,802 & 1,1010 & 0,757 & 0,825 & 1,0893 \\
\hline 32 & 0,666 & 0,751 & 1,1270 & 0,682 & 0,764 & 1,1204 \\
\hline 40 & 0,610 & 0,703 & 1,1515 & 0,623 & 0,714 & 1,1459 \\
\hline 48 & 0,575 & 0,671 & 1,1678 & 0,563 & 0,660 & 1,1734 \\
\hline 72 & 0,437 & 0,540 & 1,2346 & 0,444 & 0,547 & 1,2310 \\
\hline
\end{tabular}
las distintas fracciones molares correspondientes a los distintos tiempos de calentamiento. Todos estos valores se muestran en la Tabla I, junto con las concentraciones de TNA medidas.

Tabla I

El ajuste a esta cinética de descomposición tiene por ecuación

$$
f_{a}=0,991 \exp \left(-\frac{t}{120}\right)
$$

con un tanto por ciento de error standard del ajuste de $\mathrm{SPE}=0,82 \%$. Su representación gráfica se muestra en la figura 1.

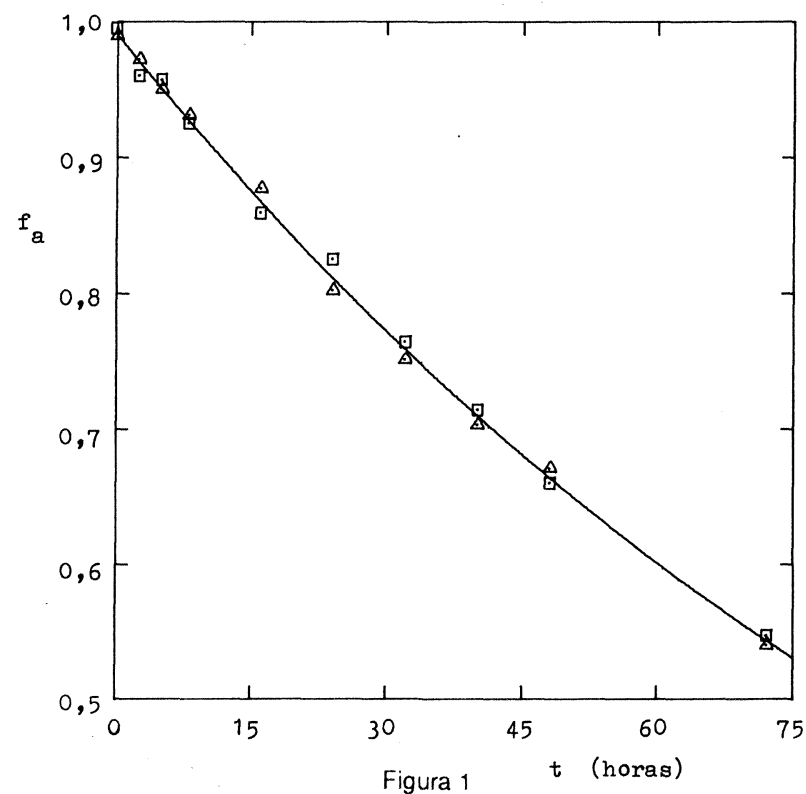


El alto grado de ajuste encontrado justifica las suposiciones que se hicieron cuando se dijo que tanto al aceite, como a los distintos productos de descomposición, se les podía asignar un peso molar medio.

El grado de alteración del aceite al sufrir el calentamiento queda así definido por su fracción molar. El peso molar medio de las distintas muestras estará definido entonces por la expresión

$$
P=f_{a} P_{a}+\left(1-f_{a}\right) P_{d}
$$

pero ya se ha indicado que no se ha determinado $P_{\mathrm{a}}$. Así se ha definido un peso molar medio, de las distintas muestras, relativo al peso molar del aceite

$$
P_{r}=\frac{P}{P_{a}}=f_{a}+1,51\left(1-f_{a}\right)
$$

cuyos valores se muestran también en la Tabla l.

Los valores molares que se calculen con las distintas magnitudes medidas estarán relativizadas al peso molar del aceite. Es decir, todas estarán divididas por un número constante. Esta circunstancia, hace imposible conocer los valores absolutos de las distintas magnitudes molares, sin embargo, su variación con respecto a otras variables, sí se pueden establecer, apareciendo los parámetros que definan estas relaciones, afectadas por dicho peso molar del aceite.

\section{b) Medida de volumen específico}

Se ha medido el volumen específico (determinado por el valor inverso de la densidad), en un intervalo de temperaturas comprendido entre 5 y $95^{\circ} \mathrm{C}$, observándose que existe una relación lineal entre el volumen específico y la temperatura.

Se ha ajustado la serie de medidas de cada muestra a la ecuación lineal de dilatación en volumen

$$
v=v_{20}(1+\alpha(T-293,2))
$$

donde $\mathrm{v}_{20}$ es el volumen específico a $20^{\circ} \mathrm{C}$. (Se ha tomado esta temperatura de referencia, como la del ambiente del laboratorio), $\alpha$ es el coeficiente de dilatación en volumen y $\mathrm{T}$ la temperatura.
Se ha comprobado que todos los coeficientes de dilatación calculados en cada muestra, solapan entre sí sus márgenes de error, lo que se ha interpretado en el sentido de que su valor permanece constante a lo largo de todo el período de calentamiento del aceite. Por lo tanto se ha procedido a calcular dicho coeficiente con los datos de todas las muestras, de ambas series, en conjunto. El valor encontrado ha sido

$$
\alpha=(7,61 \pm 0,05) \cdot 10^{-4} \mathrm{~K}^{-1}
$$

Los valores del volumen específico a $20^{\circ} \mathrm{C}$, así como los valores correspondientes a cada ajuste del "tanto por ciento de error standard" se muestran en la Tabla II donde también se incluyen los valores del volumen molar relativizado a $20^{\circ} \mathrm{C}$

\section{c) Medida de viscosidad dinámica}

En todas las muestras de ambas series, se ha comprobado el comportamiento reológico newtoniano de las mismas, mediante el reograma correspondiente (relación: esfuerzo cortante - velocidad de cizalla). Comprobándose además que la ordenada en el origen es nula. Este reograma se ha obtenido a una temperatura comprendida entre 5 y $10^{\circ} \mathrm{C}$, admitiéndose que el comportamiento newtoniano de todos los aceites se mantiene a temperaturas superiores.

1) Relación viscosidad dinámica-temperatura.- Se han propuesto numerosas ecuaciones para relacionar la viscosidad dinámica con la temperatura pero son las de tipo exponencial las que mejor se han adaptado, J. Guzmán (3) y E.N. da C. Andrade (4) establecieron que

$$
\eta=A \exp (E / R T)
$$

donde $\mathrm{A}$ y $\mathrm{E}$ son dos constantes características del líquido y $R$ la constante universal de los gases perfectos. A representa la viscosidad que tendría el líquido a una temperatura muy alta, en el supuesto de que no existiese cambio de estado y $E$ es la llamada "energía de acti-

\begin{tabular}{|c|c|c|c|c|c|c|c|}
\hline \multicolumn{4}{|c|}{ Serie 1} & \multicolumn{4}{|c|}{ Serie 2} \\
\hline$f_{a}$ & $v_{20}$ & SPE\% & $V_{120}$ & $f_{a}$ & $v_{20}$ & SPE\% & $V_{r 20}$ \\
\hline 0,989 & 1,0948 & 0,02 & 1,1009 & 0,995 & 1,0948 & 0,01 & 1,0976 \\
\hline 0,972 & 1,0925 & 0,01 & 1,1081 & 0,960 & 1,0926 & 0,03 & 1,1149 \\
\hline 0,950 & 1,0914 & 0,03 & 1,1192 & 0,957 & 1,0919 & 0,02 & 1,1158 \\
\hline 0,931 & 1,0899 & 0,04 & 1,1283 & 0,925 & 1,0893 & 0,04 & 1,1310 \\
\hline 0,877 & 1,0855 & 0,02 & 1,1536 & 0,859 & 1,0842 & 0,03 & 1,1622 \\
\hline 0,802 & 1,0813 & 0,06 & 1,1905 & 0,825 & 1,0821 & 0,05 & 1,1787 \\
\hline 0,751 & 1,0779 & 0,05 & 1,2148 & 0,764 & 1,0791 & 0,04 & 1,2090 \\
\hline 0,703 & 1,0749 & 0,06 & 1,2377 & 0,714 & 1,0755 & 0,08 & 1,2324 \\
\hline 0,671 & 1,0729 & 0,07 & 1,2529 & 0,660 & 1,0731 & 0,06 & 1,2592 \\
\hline 0,540 & 1,0658 & 0,05 & 1,3158 & 0,547 & 1,0664 & 0,07 & 1,3128 \\
\hline
\end{tabular}
vación del flujo viscoso".

El concepto de esta energía de activación se comprende fácilmente si se admite la hipótesis propuesta por

Tabla II 
Eyring (5) para explicar la viscosidad de los líquidos, mediante la aplicación de la teoría de la velocidad de la reacción química a los procesos de transporte de momento. Eyring admite que las moléculas se desplazan entre sí dentro de capas líquidas paralelas, siempre que su energía supere el valor de la "energía molecular de activación del flujo viscoso". Esta corresponde a la energía que una molécula necesita adquirir antes de que pueda sobrepasar la barrera de potencial que le impide situarse en otra posición estable dentro de la capa líquida.

En 1952, Gutmann y Simmons (6) encontraron que la energía de activación del flujo viscoso dependía de la temperatura de la forma

$$
E=E_{0} /\left[1-T_{0} / T\right]
$$

donde $E_{0}$ es la componente de la energía de activación que no depende de la temperatura y $T_{0}$ la temperatura a la cual la energía de activación se haría tan grande que el líquido no podría fluir, en el supuesto de que no hubiese cambio de estado al alcanzar esa temperatura.

Así la ecuación que relaciona la viscosidad dinámica con la temperatura toma la forma

$$
\eta=A \exp \left[E_{0} / R\left(T-T_{0}\right)\right]
$$

fórmula que coincide con la establecida por Fulcher en 1925 (7) y por Tamman y Hesse en 1926 (8) y V. Flores y Col. (9) donde ellos llamaron $B, a E_{o} / R$.

El valor del parámetro $A$ se deduce, en el ajuste por extrapolación, ya que es imposible su medida. Sin embargo se puede transformar la ecuación introduciendo un valor conocido de la viscosidad.

$$
\eta_{20}=A \exp \left[E_{0} / R\left(293,2-T_{0}\right)\right]
$$

donde $\eta_{20}$ es el valor de la viscosidad a $20^{\circ} \mathrm{C}$.

Dividiendo ambas ecuaciones se obtiene

$$
\eta=\eta_{20} \exp \left[\frac{E_{0}}{R}\left(\frac{1}{T-T_{0}}-\frac{1}{293,2-T_{0}}\right)\right]
$$

A esta ecuación se han ajustado los valores de la viscosidad medidos.

En todos los valores de $T_{0}$ encontrados en los ajustes se ha observado que sus márgenes de error se solapan entre sí, siendo éstos del orden del $5 \%$. Con el fin de adoptar un criterio de uniformidad se ha supuesto que el valor de $T_{0}$ es el mismo para todos los aceites de ambas series. Por ello se ha procedido al cálculo de $T_{0}$ con los valores de todas las muestras en conjunto. Este cálculo ha dado un valor de $T_{0}=161,1 \mathrm{~K}$.

Los valores correspondientes a la viscosidad a $20^{\circ} \mathrm{C}$ así como los valores de la energía de activación del flujo viscoso a $20^{\circ} \mathrm{C}$ y la parte de esta energía independiente de la temperatura se muestran en la Tabla III. También se incluyen los valores del tanto por ciento de error standard de los ajustes en los que se observa que el valor máximo es del orden del $1 \%$.

2) Relación viscosidad dinámica-volumen.- La relación de la viscosidad con el volumen específico se define en la literatura sobre este tema por las fórmulas de Batschinski (10) y más modernamente por la de Hildebrand (11), que establecen una relación lineal entre la fluidez (valor inverso de la viscosidad) y el volumen especifico y el volumen molar respectivamente y la de Doolittle (12), que es de tipo exponencial. Los aceites se ajustan a esta última ecuación, que por otra parte, tiene un campo de aplicación más general que las fórmulas anteriores (13).

La ecuación de Doolittle es

$$
\eta=A \exp \left[b /\left(v-v_{0}\right)\right]
$$

donde $A, b$ y $v_{0}$ son tres parámetros a determinar en el ajuste. A, tiene el mismo significado que el correspondiente en la relación viscosidad dinámica-temperatura, $v$ es el llamado "volumen específico ocupado" y expresa el volumen que tiene el líquido al bajar la temperatura, suponiendo, que no exista cambio de estado, y que debido a la compactibilidad de éste no se pueden producir movimientos de cizalla entre sus moléculas. La diferencia

\begin{tabular}{|c|c|c|c|c|c|c|c|c|c|c|c|}
\hline \multirow[b]{2}{*}{$f_{a}$} & \multicolumn{4}{|c|}{ Serie 1} & \multirow[b]{2}{*}{$\eta_{50}$} & \multicolumn{6}{|c|}{ Serie 2} \\
\hline & $\eta_{20}$ & $E_{0}$ & $E_{20}$ & SPE\% & & $f_{a}$ & $\eta_{20}$ & $E_{。}$ & $E_{20}$ & SPE\% & $\eta_{50}$ \\
\hline 0,989 & 80,28 & $1.666,8$ & $3.699,5$ & 1,03 & 25,03 & 0,995 & 80,02 & $1.669,7$ & $3.706,0$ & 0,77 & 25,03 \\
\hline 0,972 & 86,22 & $1.687,9$ & $3.746,3$ & 0,78 & 26,23 & 0,960 & 86,04 & $1.700,4$ & $3.774,1$ & 0,40 & 26,27 \\
\hline 0,950 & 92,24 & $1.698,6$ & $3.770,1$ & 0,47 & 27,77 & 0,957 & 91,34 & $1.711,9$ & $3.799,6$ & 0,43 & 27,33 \\
\hline 0,931 & 101,5 & $1.719,3$ & $3.816,0$ & 1,08 & 29,93 & 0,925 & 101,8 & $1.734,1$ & $3.849,0$ & 0,70 & 30,12 \\
\hline 0,877 & 125,3 & $1.770,8$ & $3.930,3$ & 0,62 & 35,96 & 0,850 & 124,1 & $1.788,2$ & $3.968,9$ & 0,62 & 35,27 \\
\hline 0,802 & 146,5 & $1.824,1$ & $4.048,7$ & 0,43 & 40,54 & 0,825 & 144,9 & $1.815,0$ & $4.028,5$ & 0,61 & 39,77 \\
\hline 0,751 & 170,2 & $1.874,1$ & $4.159,6$ & 0,66 & 46,27 & 0,764 & 168,4 & $1.867,2$ & $4.144,3$ & 0,72 & 44,81 \\
\hline 0,703 & 208,5 & $1.905,9$ & $4.230,2$ & 0,80 & 54,40 & 0,714 & 202,1 & $1.912,7$ & $4.245,4$ & 0,55 & 53,02 \\
\hline 0,671 & 232,1 & $1.941,0$ & $4.308,0$ & 0,14 & 59,12 & 0,660 & 232,0 & $1.955,7$ & $4.340,7$ & 0,45 & 59,12 \\
\hline 0,540 & 350,3 & $2.056,4$ & $4.564,1$ & 0,96 & 82,19 & 0,547 & 352,1 & $2.068,1$ & $4.590,1$ & 0,76 & 83,19 \\
\hline
\end{tabular}
$\left(v-v_{0}\right)$ es el llamado "volumen libre" o "volumen vacio". Por último, b es un parámetro característico del líquido, que tiene dimensión de volumen.

Tabla III 
Como en el caso de la relación viscosidad-temperatura, se puede eliminar el parámetro $A$ en función del valor de la viscosidad para un cierto valor del volumen específico, así

$$
\eta=\eta_{20} \exp \left[b\left(\frac{1}{v-v_{0}}-\frac{1}{v_{20}-v_{0}}\right)\right]
$$

siendo $\eta_{20}$ y $v_{20}$, la viscosidad y el volumen específico a $20^{\circ} \mathrm{C}$ de temperatura.

A esta ecuación se puede llegar partiendo de la (4) que expresa la relación viscosidad-temperatura, si existe una relación lineal entre el volumen y la temperatura.

Ya se ha comprobado que existe esa relación, es la definida por la ecuación (3). Es fácil, pues, deducir que

$$
b=\frac{E_{0} \alpha v_{20}}{R} \quad y \quad v_{0}=v_{20}\left(1+\alpha\left(T_{0}-293,2\right)\right)
$$

Como comprobación se han calculado los valores de b y de $v_{0}$, así como, el "tanto por ciento de error standard", realizado con estos valores de los parámetros y las medidas, los cuales se muestran en la Tabla IV. Se observa que el error máximo obtenido está próximo al $2 \%$, ello es debido a que tanto $b$, como $v_{0}$, están calculados con parámetros que proceden de dos ajustes independientes entre sí, sin embargo, este error es inferior al que se comete al efectuar las medidas de viscosidad dinámica.

\section{d) Medida de índice de refracción}

Se ha medido el índice de refracción, en un intervalo de temperaturas comprendido entre 10 y $70^{\circ} \mathrm{C}$, observándose que existe una variación lineal entre el índice de refracción y la temperatura, ajustándose a la ecuación

$$
\mathrm{n}=\mathrm{n}_{20}(1+C(T-293,2))
$$

donde $n_{20}$ es el índice de refracción a $20^{\circ} \mathrm{C}$, y C el "coeficiente de variación térmica", definido como la variación del índice de refracción por unidad de temperatura y de indice de refracción.

\begin{tabular}{|c|c|c|c|c|c|c|c|}
\hline \multicolumn{4}{|c|}{ Serie 1} & \multicolumn{4}{|c|}{ Serie 2} \\
\hline$f_{a}$ & b & SPE\% & $v_{0}$ & $f_{a}$ & b & SPE\% & $v_{0}$ \\
\hline 0,989 & 0,6932 & 1,11 & 0,9847 & 0,995 & 0,6912 & 1,80 & 0,9847 \\
\hline 0,972 & 0,7062 & 1,97 & 0,9827 & 0,960 & 0,7042 & 1,12 & 0,9828 \\
\hline 0,950 & 0,7118 & 1,24 & 0,9817 & 0,957 & 0,7156 & 1,43 & 0,9821 \\
\hline 0,931 & 0,7231 & 1,84 & 0,9803 & 0,925 & 0,7205 & 1,79 & 0,9798 \\
\hline 0,877 & 0,7361 & 1,60 & 0,9764 & 0,859 & 0,7408 & 1,45 & 0,9752 \\
\hline 0,802 & 0,7545 & 1,81 & 0,9726 & 0,825 & 0,7600 & 1,56 & 0,9733 \\
\hline 0,751 & 0,7626 & 1,69 & 0,9695 & 0,764 & 0,7760 & 1,46 & 0,9706 \\
\hline 0,703 & 0,7845 & 1,63 & 0,9668 & 0,714 & 0,7817 & 1,45 & 0,9674 \\
\hline 0,671 & 0,7970 & 1,32 & 0,9658 & 0,660 & 0,7970 & 1,44 & 0,9652 \\
\hline 0,540 & 0,8393 & 1,88 & 0,9587 & 0,547 & 0,8357 & 1,63 & 0,9592 \\
\hline
\end{tabular}

Se ha comprobado que todos los "coeficientes de va-

Tabla IV

riación térmica" calculados con cada muestra, solapan entre sí sus márgenes de error, lo que se ha interpretado, que su valor no varía con los distintos tiempos de calentamiento. Por ello se ha procedido a calcular el coeficiente de variación térmica con los datos de los aceites, de ambas series, en conjunto. El valor hallado es

$$
\mathrm{C}=(-2,32 \pm 0,07) \cdot 10^{-4} \mathrm{~K}^{-1}
$$

Los valores del índice de refracción a $20^{\circ} \mathrm{C}$, así como los valores correspondientes a cada ajuste del "tanto por ciento de error standard" se muestran en la Tabla V.

\section{e) Medidas de la temperatura y de la entalpia de} fusión

Con un calorímetro diferencial de barrido se han medido la temperatura y la entalpía específica de fusión. Previamente se ha cristalizado el aceite siguiendo un barrido de temperaturas, a una velocidad de $5^{\circ} \mathrm{C}$ por minuto hasta $-80^{\circ} \mathrm{C}$, en esta temperatura se le ha mantenido durante 10 minutos, para tener la seguridad de que está cristalizado en forma $\alpha$. Después siguiendo a la misma velocidad de barrido, se le ha calentado hasta $30^{\circ} \mathrm{C}$, a fin de borrar toda la posible influencia del proceso seguido. Se ha observado lo siguiente:

1. En la cristalización.- A una temperatura comprendida entre $-14 \pm 2^{\circ} \mathrm{C}$ (ver fig. 2), en todos los aceites, aparece una transición vítrea, que hace descender el calor específico, en $0,1 \mathrm{Cal} / \mathrm{g}$, esta disminución se mantiene hasta que alcanza su temperatura de cristalización. Parece como si para cristalizar tuviera que pasar por una configuración de los TNA y productos de descomposición menos energética. Junto con la transición vítrea, aparece un pequeño pico que posiblemente sea la entalpía necesaria para llegar a ese estado menos energético.

Este pico va creciendo conforme crece la alteración, llegando a un valor máximo, cuando la fracción molar de los TNA es del orden de 0,8 (ver fig. 3), donde se ha representado el valor de la entalpía específica de este pico en función de la fracción molar de los TNA. Pasado este valor el pico vuelve a disminuir hasta alcanzar un valor del orden de los iniciales. 
Tabla V

\begin{tabular}{|c|c|c|c|c|c|c|c|c|c|}
\hline $\mathbf{f}_{\mathbf{a}}$ & $\mathrm{n}_{20}$ & $\begin{array}{l}\text { Serie } 1 \\
\text { SPE\% }\end{array}$ & $R_{r}$ & $V_{\text {ro }}$ & $f_{a}$ & $\mathrm{n}_{20}$ & $\begin{array}{l}\text { Serie } 2 \\
\text { SPE\% }\end{array}$ & $R_{r}$ & $V_{r o}$ \\
\hline 0,989 & 1,4691 & 0,04 & 0,3066 & 0,9903 & 0,995 & 1,4689 & 0,03 & 0,3056 & 0,9873 \\
\hline 0,972 & 1,4696 & 0,03 & 0,3089 & 0,9967 & 0,960 & 1,4697 & 0,02 & 0,3108 & 1,0028 \\
\hline 0,950 & 1,4697 & 0,03 & 0,3121 & 1,0067 & 0,957 & 1,4696 & 0,02 & 0,3111 & 1,0037 \\
\hline 0,931 & 1,4699 & 0,05 & 0,3147 & 1,0148 & 0,925 & 1,4703 & 0,07 & 0,3157 & 1,0173 \\
\hline 0,877 & 1,4711 & 0,07 & 0,3225 & 1,0376 & 0,859 & 1,4711 & 0,03 & 0,3249 & 1,0453 \\
\hline 0,802 & 1,4718 & 0,07 & 0,3332 & 1,0708 & 0,825 & 1,4716 & 0,02 & 0,3298 & 1,0602 \\
\hline 0,751 & 1,4725 & 0,06 & 0,3404 & 1,0927 & 0,764 & 1,4722 & 0,02 & 0,3386 & 1,0874 \\
\hline 0,703 & 1,4730 & 0,08 & 0,3472 & 1,1133 & 0,714 & 1,4729 & 0,03 & 0,3456 & 1,1085 \\
\hline 0,671 & 1,4735 & 0,12 & 0,3518 & 1,1270 & 0,660 & 1,4737 & 0,05 & 0,3536 & 1,1326 \\
\hline 0,540 & 1,4752 & 0,10 & 0,3706 & 1,1836 & 0,547 & 1,4750 & 0,08 & 0,3696 & 1,1808 \\
\hline
\end{tabular}

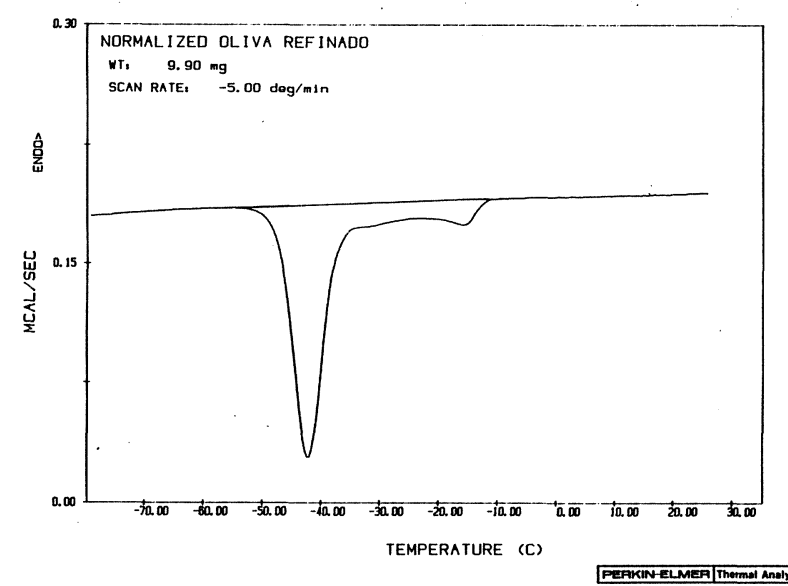

Figura 2

2. En la fusión.- Iniciado el calentamiento a partir de $-80^{\circ} \mathrm{C}$ (ver fig. 4), el aceite mantiene constante su calor específico hasta poco antes de iniciar su fusión en que aparece la transición vítrea, disminuyendo el calor específico en el mismo valor que en la cristalización, y apareciendo el pequeño pico exotérmico, que se observó en la cristalización iniciándose inmediatamente la fusión. Es decir, que para fundir, al igual que para cristalizar, el aceite, tanto alterado como no alterado, pasa por un estado menos energético.

Las temperaturas de fusión, las entalpías específicas de fusión $\mathrm{H}_{e}$ y las exotérmicas del pequeño pico $H_{g}$ se muestran en la Tabla VI.

\section{DISCUSION DE LOS RESULTADOS}

Se han relacionado los distintos parámetros deducidos de los ajustes de las ecuaciones que los definen, con la alteración producida por la termooxidación del aceite, valorada por la fracción molar de TNA.

Las diferentes muestras son una mezcla de una cierta cantidad de aceite, más otra de los productos de descom-

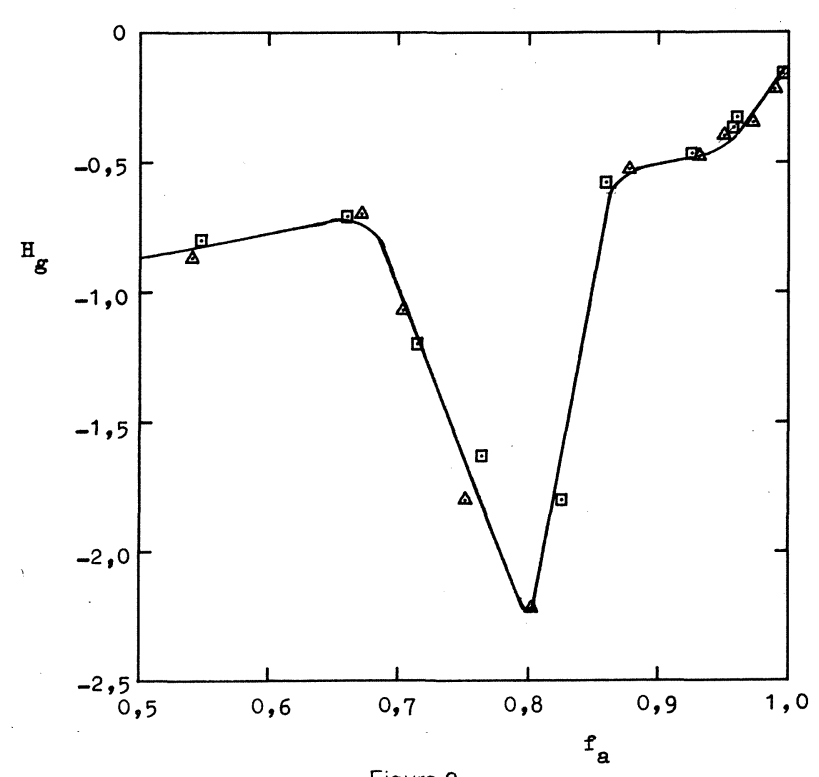

Figura 3

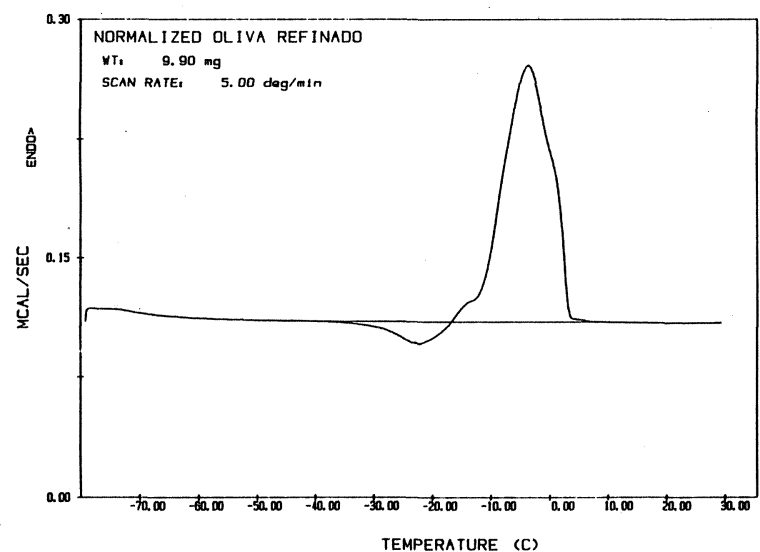

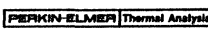

Figura 4 
Tabla VI

\begin{tabular}{|c|c|c|c|c|c|c|c|}
\hline \multicolumn{4}{|c|}{ Serie 1} & \multicolumn{4}{|c|}{ Serie 2} \\
\hline $\mathbf{f}_{\mathrm{a}}$ & $\mathbf{T}$ & $\mathrm{H}_{\mathrm{e}}$ & $\mathrm{H}_{\mathrm{g}}$ & $f_{a}$ & $\mathbf{T}$ & $\mathrm{H}_{\mathrm{e}}$ & $\mathrm{H}_{\mathrm{g}}$ \\
\hline 0,989 & 261,7 & 18,0 & $-0,22$ & 0,995 & 262,5 & 18,4 & $-0,16$ \\
\hline 0,972 & 260,5 & 16,9 & $-0,35$ & 0,960 & 260,4 & 16,7 & $-0,33$ \\
\hline 0,950 & 259,9 & 16,2 & $-0,40$ & 0,957 & 260,7 & 16,3 & $-0,37$ \\
\hline 0,931 & 258,4 & 15,4 & $-0,48$ & 0,925 & 258,9 & 15,2 & $-0,47$ \\
\hline 0,877 & 256,8 & 13,5 & $-0,53$ & 0,859 & 255,9 & 13,5 & $-0,58$ \\
\hline 0,802 & 251,6 & 12,2 & $-2,22$ & 0,825 & 252,4 & 12,4 & $-1,80$ \\
\hline 0,751 & 248,5 & 11,2 & $-1,80$ & 0,764 & 250,0 & 11,3 & $-1,63$ \\
\hline 0,703 & 247,7 & 10,3 & $-1,07$ & 0,714 & 247,9 & 10,8 & $-1,20$ \\
\hline 0,671 & 248,2 & 9,42 & $-0,70$ & 0,660 & 248,0 & 9,89 & $-0,71$ \\
\hline 0,540 & 248,1 & 6,40 & $-0,87$ & 0,547 & 248,5 & 6,63 & $-0,80$ \\
\hline
\end{tabular}

posición. Siguiendo las leyes de mezclas (14), si el parámetro varía siguiendo una relación lineal con la fracción molar, se dice que sigue una relación "ideal" y en el caso contrario "no ideal".

El intervalo de fracciones molares de TNA en el que se han realizado medidas varía entre 1,0 y 0,5 . Así un comportamiento "ideal" estará definido por la recta que tiene su origen $y$ fin en los puntos $\left(1,0 ; X_{1}\right)$ y $\left(0,5 ; X_{2}\right)$ siendo $X_{1}$ y $X_{2}$ los valores del parámetro en estudio.

Esta recta tiene por ecuación

$$
X=\left(2 f_{a}-1\right) X_{1}+2\left(1-f_{a}\right) X_{2}
$$

\section{a) Volumen}

El volumen molar a $20^{\circ} \mathrm{C}$ relativizado cuyos valores se muestran en la Tabla II sigue una variación lineal definida por la recta

$$
V_{r 20}=\left(2 f_{a}-1\right) V_{1}+2\left(1-f_{a}\right) V_{2}
$$

donde $V_{1}$ y $V_{2}$ tienen los valores de 1,0951 y 1,3353 respectivamente. El tanto por ciento de error standard de:

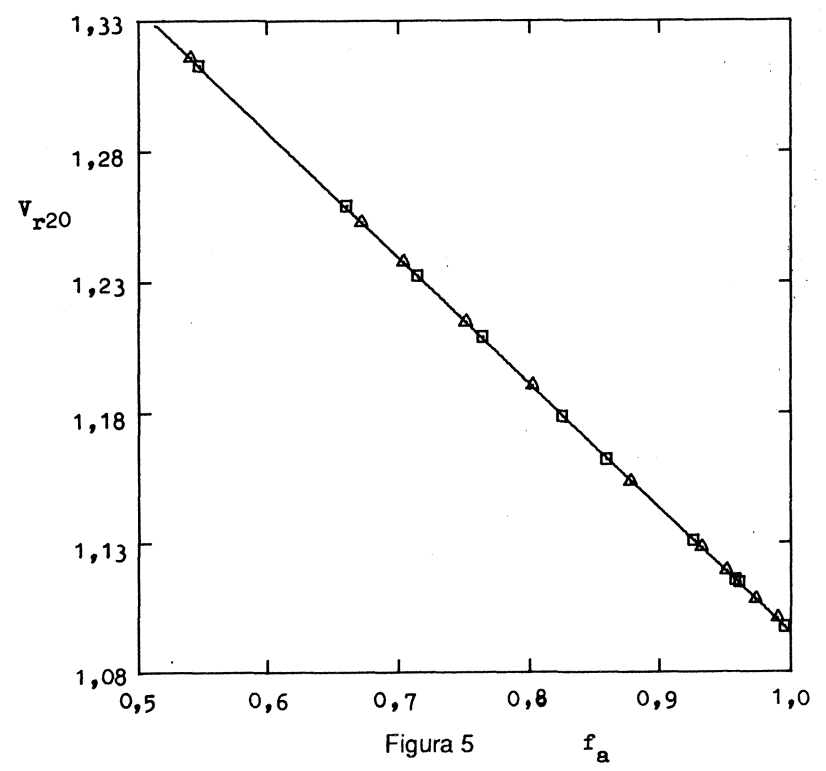

este ajusta es SPE $=0,33 \%$. Su representación gráfica se muestra en la figura 5.

Es evidente que si el volumen molar sigue una ley lineal, lo que suele ser norma general en los líquidos, su volumen específico a $20^{\circ} \mathrm{C}$ no la seguirá, es fácil comprobar que su variación es

$$
v_{20}=\frac{V_{r 20}}{P_{r}}=\frac{\left(2 f_{a}-1\right) V_{1}+2\left(1-f_{a}\right) V_{2}}{f_{a}+1,51\left(1-f_{a}\right)}
$$

cuya representación gráfica se muestra en la figura 6 . El tanto por ciento de error standard calculado es SPE = $0,33 \%$.

El valor en exceso definido por la diferencia entre el valor obtenido y el valor dado por la recta ideal (ver fig. 7) es

$$
v_{20}^{E}=\frac{\left(2 f_{a}-1\right) V_{1}+2\left(1-f_{a}\right) V_{2}}{f_{a}+1,51\left(1-f_{a}\right)}-\left(\left(2 f_{a}-1\right) v_{1}+2\left(1-f_{a}\right) v_{2}\right)
$$

donde $v_{1}$ y $v_{2}$ son los valores del volumen específico a $20^{\circ} \mathrm{C}$ para una fracción molar de 1 y 0,5 y tienen un valor de 1,0951 y 1,0640 respectivamente.

Se observa que el volumen específico en exceso tiene un mínimo dentro del intervalo de fracción molar comprendido entre 0,7 y 0,8 .

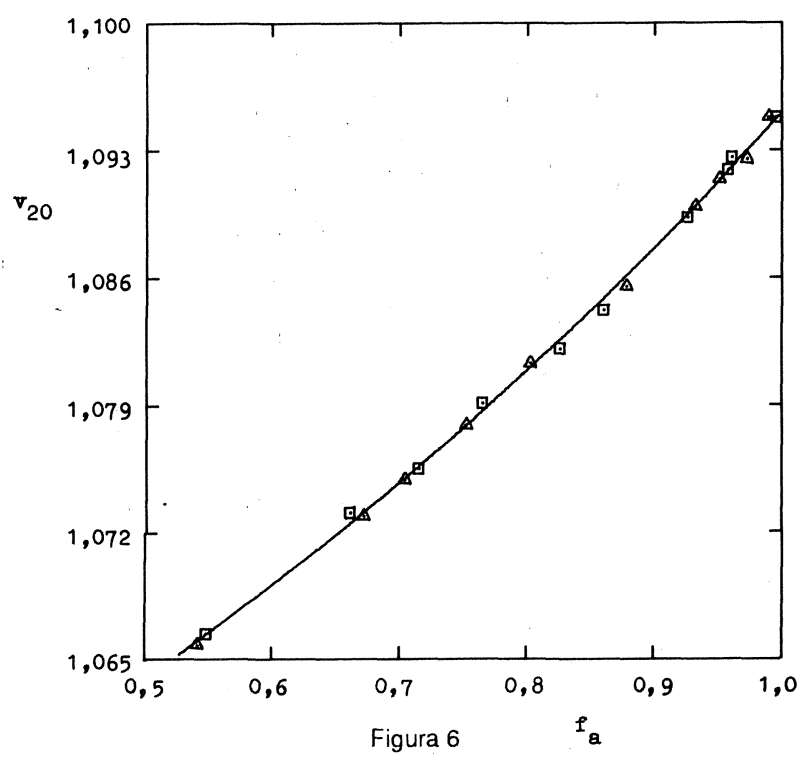




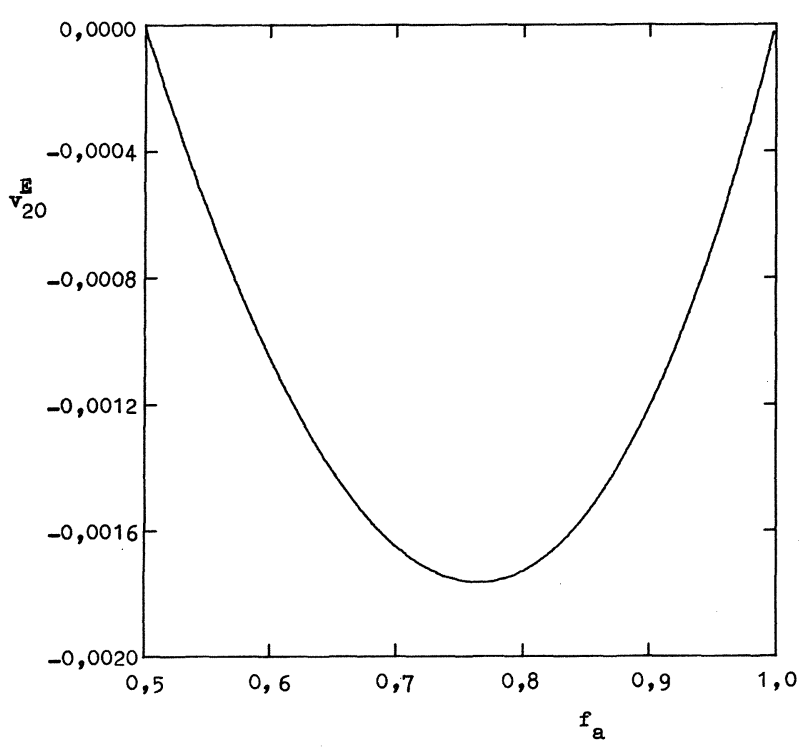

Figura 7

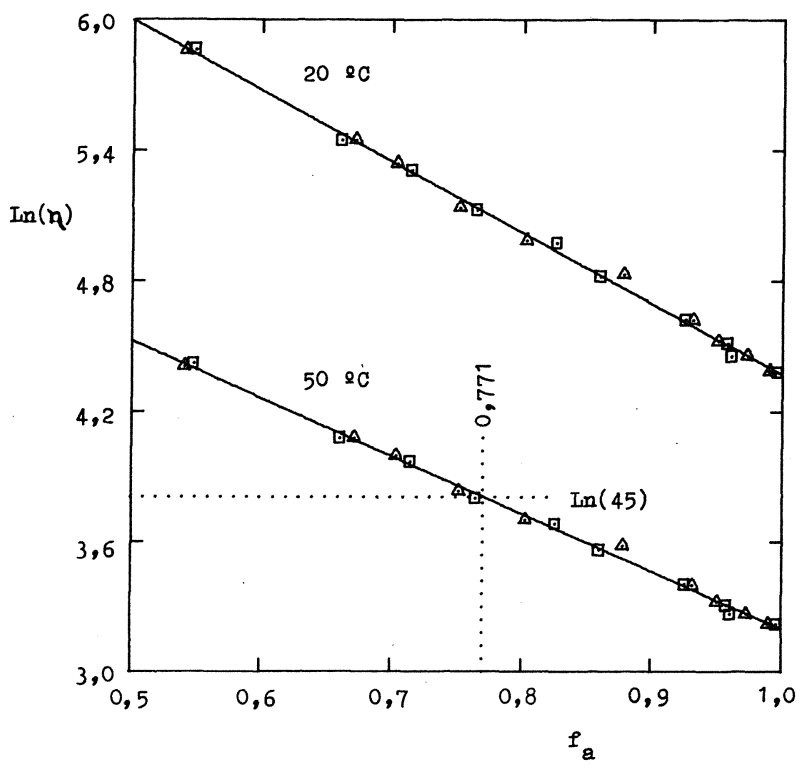

Figura 8

\section{b) Viscosidad dinámica}

1. Relación viscosidad-temperatura.- La viscosidad de mezclas de aceite con los productos de descomposición termooxidativa sigue la clásica ley establecida por Arrhenius en 1887 (15), en la que el logaritmo de la viscosidad de la mezcla es función lineal de los logaritmos de las viscosidades de los componentes y la fracción molar de los TNA.

Esta relación dentro del intervalo de fracción molar medido es

$$
\operatorname{Ln}\left(\eta_{m}\right)=\left(2 f_{a}-1\right) \operatorname{Ln}\left(\eta_{1}\right)+2\left(1-f_{a}\right) \operatorname{Ln}\left(\eta_{2}\right)
$$

donde $\eta_{m}, \eta_{1}$ y $\eta_{2}$ son las viscosidades de la mezcla, y del aceite para una fracción molar $f_{a}=1$ y $f_{a}=0,5$ respectivamente, cuyos logaritmos tienen un valor de 4,3678 y 5,9915 respectivamente, con un tanto por ciento de error standard $\mathrm{SPE}=0,51 \%$, la representación gráfica se muestra en la figura 8.

También en esta misma figura se incluye la recta que define la mezcla a $50^{\circ} \mathrm{C}$ cuyos valores de la viscosidad se incluyen en la Tabla III.

R.E. Timms en un trabajo publicado en 1985 (16) dice que los aceites usados en la fritura de alimentos, deben ser rechazados cuando su viscosidad a $50^{\circ} \mathrm{C}$ alcance un valor de $45 \mathrm{mPa}$.s. La viscosidad alcanza este valor para una fracción molar $f_{a}=0,771$.

En la figura 9, se muestra la variación de la energía de activación del flujo viscoso a $20^{\circ} \mathrm{C}$. Se observa que ésta es proporcional al peso molar de la mezcla. El valor de la constante de proporcionalidad es $3696 \pm 6 \mathrm{Cal} / \mathrm{mol}$, la cual, al estar relativizado el peso molar de la mezcla, es el producto de otra constante por el peso molar del aceite.

Del hecho de que la energía de activación sea proporcional al peso molar se deduce que para que se produzcan movimientos de cizalla, se necesita una energía media por unidad de masa constante para todas las muestras y solo depende ésta de la temperatura.

2. - Relación viscosidad-volumen específico.- En la relación viscosidad-volumen específico, el parámetro b, al ser proporcional al volumen específico a $20^{\circ} \mathrm{C}$, tendrá una variación del mismo tipo que éste por lo que no tiene interés su estudio. El parámetro importante en esta relación es la variación del volumen específico ocupado.

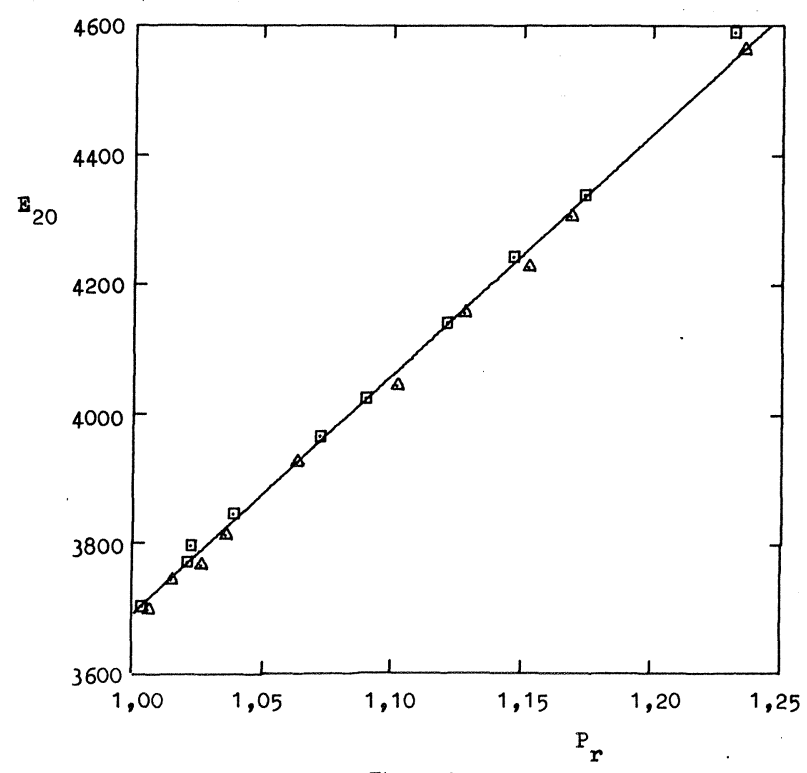

Figura 9 
Doolittle estudiando hidrocarburos saturados encontró una variación de tipo exponencial en función del peso molar de la forma,

$$
\mathrm{V}_{\mathrm{o}}=\exp [10 / \mathrm{P}]
$$

Aquí se ha encontrado que esta variable es

$$
v_{o}=0,855 \exp \left[0,141 / P_{d}\right]
$$

con un tanto por ciento de error standard SPE $=0,034 \%$ (ver fig. 10).

Si se comparan con los datos de los artículos de Doolittle, las relaciones $v_{0} / 1$ y $10 / P$, con los de este trabajo $v_{0} / 0,855$ y $0,141 / P_{r}$, éstos últimos son equivalentes a los correspondientes de Doolittle para el hexano. Se podría pensar que la ordenación de las cadenas ácidas de los triglicéridos, que en su mayoría son de 18 carbonos, se empaqueten de la misma forma que tres cadenas de hexano alineadas.

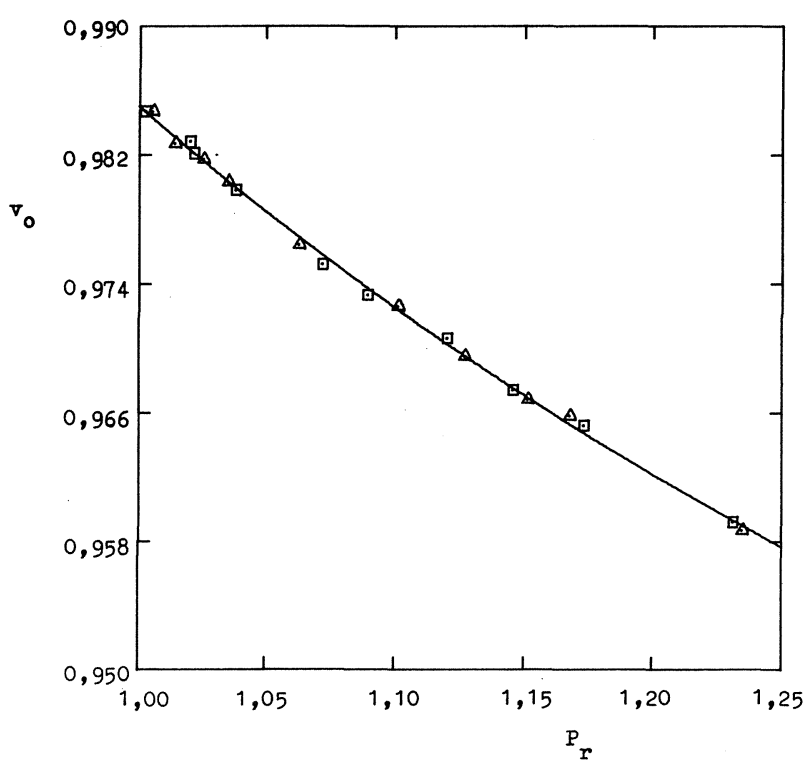

Figura 10

\section{c) Refracción molar}

Una función interesante en la que interviene el índice de refracción, es la llamada refracción molar, cuyos valores se dan en la Tabla $V$, y que tienen por expresión:

$$
R_{r}=\frac{n^{2}+1}{n^{2}+2} V_{r}
$$

donde $n$ es el índice de refracción y $V_{r}$ el volumen molar, medidos ambos a la misma temperatura. El valor de la refracción molar depende de la longitud de onda de la luz empleada para medir el índice de refracción y no depende de la temperatura.

Al igual que el volumen molar, la refracción molar es aditiva, como se muestra en la figura 11 . Su dependencia lineal con respecto a la fracción molar de TNA es

$$
R_{r}=\left(2 f_{a}-1\right) R_{r 1}+2\left(1-f_{a}\right) R_{r 2}
$$

donde $R_{r 1}$ y $R_{r 2}$ son las refracciones molares correspondientes a las fracciones molares 1 y 0,5 , respectivamente, y tiene los valores de 0,3050 y 0,3763 con un tanto por ciento de error standard de SPE $=0,031 \%$.

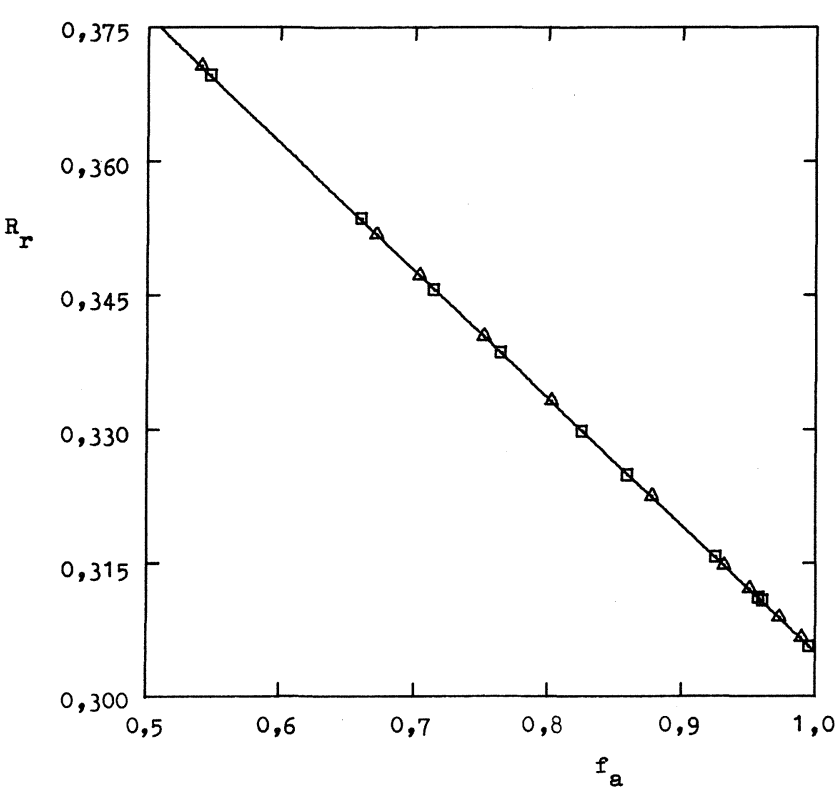

Figura 11

Se sabe que la refracción molar medida con una luz de longitud de onda muy grande mide el volumen molar ocupado (14). Por otra parte de la relación entre la viscosidad dinámica con el volumen específico, según la fórmula de Doolittle, uno de sus parámetros es el volumen específico ocupado, el cual multiplicado por el peso molar da el volumen molar ocupado, cuyos valores se muestran en la Tabla V.

Se observa que los valores del volumen molar ocupado son proporcionales a la refracción molar

$$
V_{r o}=K R_{r}
$$

siendo la constante de proporsionalidad $K=3,22 \pm 0,01$ para todas las muestras, lo que indica que los fenómenos de relajación dieléctrica (17) que se producen entre la parte baja del infrarrojo y el espectro visible se producen siempre en la misma proporción, dentro del intervalo de fracción molar de TNA que se ha estudiado.

\section{d) Entalpias especificas y temperaturas de fusion}

La representación de las medidas de entalpía de fusión en función de la fracción molar de TNA se muestran en la figura 12.

Dado que no se conoce la dependencia que existe entre dichas variables, se ha ajustado a un desarrollo en serie de potencias hasta el tercer orden, con el siguiente resultado 


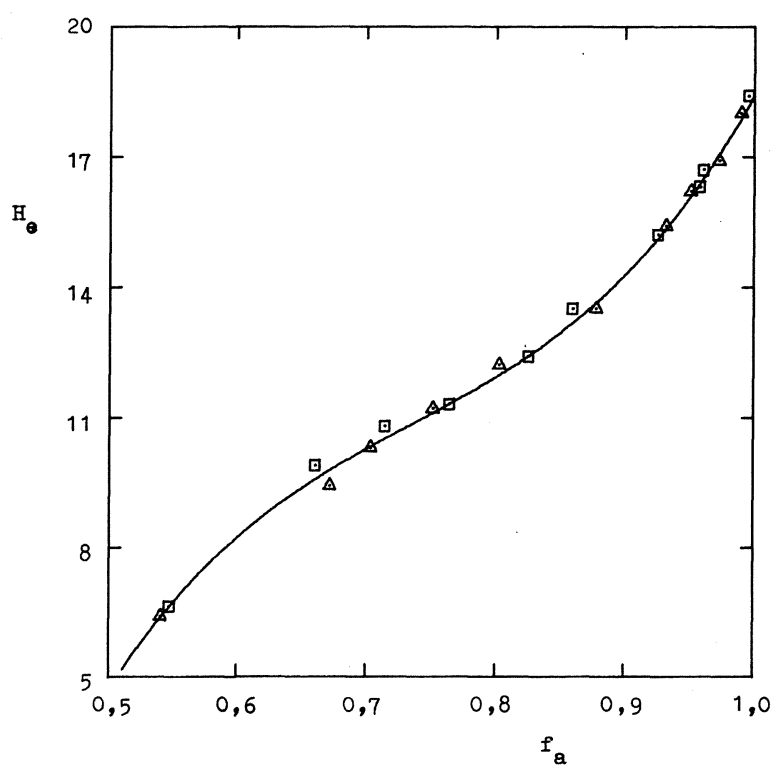

Figura 12

$$
H_{e}=-74,69+316,5\left(f_{a}\right)-407,7\left(f_{a}\right)^{2}+184,4\left(f_{a}\right)^{3}
$$

El tanto por ciento de error standard es SPE $=1,5 \%$.

En esta representación se observan dos hechos: primero que para una fracción molar de 0,737 existe un punto de inflexión, esto supone un cambio de curvatura a ambos lados de ese punto, es decir, un cambio de comportamiento de la mezcla de aceite y productos de descomposición, y segundo que para una fracción molar de 0,420 (valor extrapolado) la entalpía de fusión es nula, lo que quiere decir que a partir de ese punto la mezcla no funde y por lo tanto no cristaliza. Es decir, el contenido de productos de descomposición es tan grande, que esa mezcla es incapaz de cristalizar, como tal mezcla. Permanece en un estado líquido con una viscosidad muy alta.

Atendiendo a la temperatura de fusión, el diagrama de equilibrio de un sistema de dos componentes miscibles en estado líquido, produce un descenso en el punto de fusión del líquido puro. La relación entre la cantidad añadida y el punto de fusión, supuesta la disolución líquida ideal, tiene por ecuación

$$
\operatorname{Ln}(f)=\frac{H_{0}}{R}\left(\frac{1}{T_{0}}-\frac{1}{T}\right)
$$

donde $f, H_{0}$ y $T_{0}$, son la fracción molar del líquido, la entalpía molar y temperatura de fusión del líquido puro respectivamente.

En la figura 13 se observa que la serie de puntos cuya fracción molar es superior a 0,75 se ajustan a la ecuación anterior, siendo la forma de ésta

$$
\operatorname{Ln}\left(f_{a}\right)=1368\left(\frac{1}{262,5}-\frac{1}{T}\right)
$$

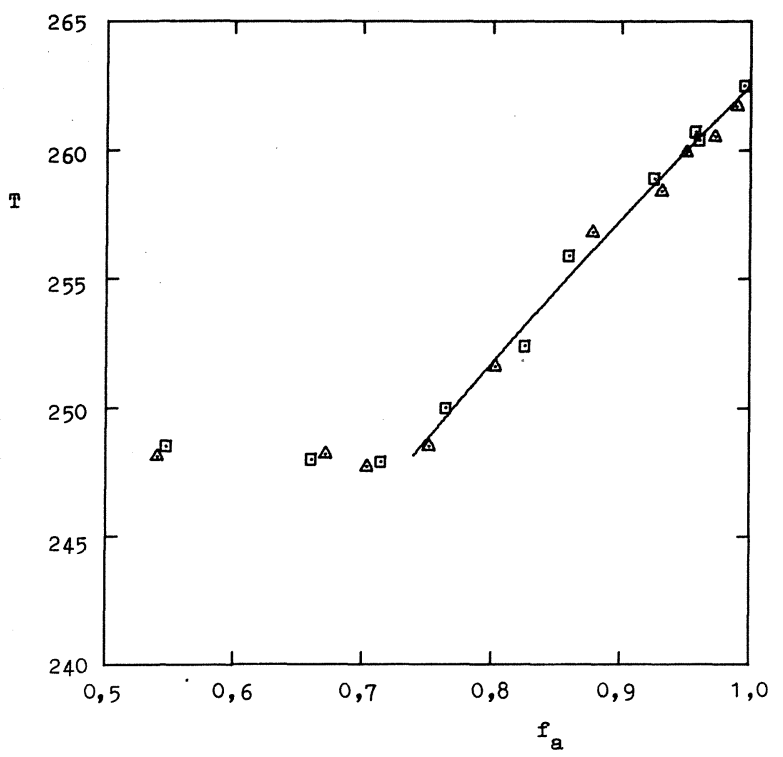

Figura 13

con un tanto por ciento de error standard SPE $=0,19 \%$. $\mathrm{El}$ valor de $\mathrm{H}_{0}$ encontrado en este ajuste tiene un valor de $2719 \mathrm{Cal} / \mathrm{mol}$. Valor mucho más pequeño del que se obtendría al multiplicar la entalpía específica de fusión del aceite medido por su peso molar. Sin embargo, no se olvide que la entalpía de fusión disminuye cuando la mezcla aumenta, y el aceite (el cual se ha definido en todo lo que respecta a este estudio como un producto "puro") es una mezcla de triglicéridos.

Con los puntos cuya fracción molar es inferior a 0,75 , y dado que la entalpía específica de fusión se anula a partir de 0,42 no se puede establecer un ajuste del tipo anterior.

Se puede decir que la mezcla de aceite y productos de descomposición termooxidativa se comporta como una mezcla eutéctica en lo que respecta al descenso de la temperatura de fusión, hasta una fracción molar de TNA de 0,75 pero sin poder definir un punto eutéctico, al no poder definir una curva similar a la anterior con los productos de descomposición.

\section{CONSIDERACION FINAL}

A lo largo de todo este estudio se ha observado que hasta que la alteración del aceite llega a una fracción molar comprendida entre 0,7 y 0,8, la mezcla de aceite y productos de descomposición se comporta como un "líquido ideal". Para fracciones molares inferiores los productos de descomposición hacen cambiar dicho comportamiento en algunas de las magnitudes medidas, tales como el volumen específico, y la entalpía y temperatura de fusión. Este cambio aparece en el mismo lugar en que Timms recomienda que se deben rechazar los aceites usados en la fritura de alimentos. 


\section{AGRADECIMIENTO}

El autor quiere hacer constar su más profunda gratitud a la Srta. Pilar García Rodríguez de Quesada, Ayudante de Investigación del Consejo Superior de Investigaciones Científicas, con destino en el Instituto de la Grasa y sus Derivados, por su desinteresada y eficaz colaboración, tanto en los trabajos de laboratorio, como en los de cálculo, redacción y mecanografía, sin cuya ayuda no habría sido posible la realización de este estudio.

\section{BIBLIOGRAFIA}

1. Dobarganes García, M.C. (1980).- "Aspectos generales de las grasas calentadas".- Grasas y Aceites 31, 417-422.

2. Waltking, A.E. y Wessels, H. (1981).- "Chromatographic separation of polar and nonpolar components of frying fats".- J. Assoc. Off. Anal. Chem. 64, 1.329-1.330.

3. Guzmán, J. (1913).- Anales de la Sociedad Española de Física y Química 11, 353.

4. Andrade, E.N. da C. (1934).- Phil. Mag. 17, 497-511 y 698-732.

5. Eyring, H. (1935).- "The activated complex in chemical reactions".J. Chem. Phys. 3, 107-115.

6. Gutmann, F. y Simmons, L.M. (1952).- "The temperature dependence of the viscosity of liquids".- J. Appl. Phys. 23, 977-978.

7. Fulcher, G.S. (1925).- J. Amer. Ceram. Soc. 8, 339

8. Tamman, G. y Hesse, W. (1926).- Z. Anorg. Allgem. Chem. 156, 245.
9. Flores Luque, V.; Cabrera Martín, J. y Gómez Herrera, C. (1982)"Variaciones de la viscosidad y de la densidad con la temperatura en aceites de oliva, girasol y soja españoles".- Grasas y Aceites 33 334-339.

10. Batschinski, A.J. (1913).- Z. Phys. Chem. 84, 643-705.

11. Hildebrand, J.H. (1977).- "Viscosity and Diffusivity".- John Wiley and Sons Inc., New York.

12. Doolittle, A.K.- "Studies in Newtonian Flow".

I. "The dependence of the viscosity of liquids on temperature".- J. Appl. Phys. 22 (1951), 1.031-1.035.

II. "The dependence of the viscosity of liquids on free-space".- J Appl. Phys. 22 (1951), 1.471-1.475.

III. "The dependence of the viscosity of liquids on molecular weight and free-space (in homologous series)".- J. Appl. Phys. 23 (1952), 236-239.

IV. "Viscosity vs molecular weight in liquids; viscosity vs concentration in polymer solutions".- J. Appl. Phys. 23 (1952), 418-426. $\mathrm{V}$. "Further verification of the free-space viscosity equation".- J. Appl. Phys. 28 (1957), 901-905

13. Flores Luque, V.; Cabrera Martin, J. y Gómez Herrera, C. (1983)."Estudios físico-químicos de miscelas de aceites vegetales. X. Relaciones entre fluidez y volumen específico en mezclas binarias de un aceite de girasol o de soja crudo y tricloroetileno o percloroetileno". Grasas y Aceites 34, 380-385.

14. Glasstone, S. (1953).- "Tratado de Química Física".- Ediciones Aguilar, S.A., Madrid.

15. Arrhenius, S.A. (1887).--Z. Physik. Chem. 1, 285

16. Timms, R.E. (1985).- "Physical properties of oils and mixtures of oils".- J. Am. Oil Chemists' Soc. 62, 241-249.

17. Diaz Peña, M. y Roig Muntaner, A. (1975).- "Química Fisica".- Editorial Alambra, Madrid.

(Recibido: Abril 1991) 